\title{
Genome-wide Investigation of Genetic Diversity in the Poultry Red Mite, Dermanyssus Gallinae, From European Farms Utilising a NGS-Multiplex Platform
}

\section{Eleanor Karp-Tatham ( $\nabla$ ekarp@rvc.ac.uk)}

RVC: The Royal Veterinary College

\section{Dong Xia}

RVC: The Royal Veterinary College

\section{Alasdair J Nisbet}

Moredun Research Institute

\section{Teresa Letra Mateus}

Development and Policies Research Center

Fiona M. Tomley

RVC: The Royal Veterinary College

\section{Damer P. Blake}

RVC: The Royal Veterinary College

\section{Research Article}

Keywords: Genetic diversity, Dermanyssus gallinae, SNPs, Population genetics, layer chickens

Posted Date: December 15th, 2021

DOI: https://doi.org/10.21203/rs.3.rs-1131116/v1

License: (c) (i) This work is licensed under a Creative Commons Attribution 4.0 International License. Read Full License 


\section{Abstract}

The poultry red mite (Dermanyssus gallinae), an obligatory blood feeding ectoparasite, is primarily associated with egg laying hens where it is estimated to cause losses of $\sim € 230$ million per annum from European farmers. Current control strategies, including the use of acaricidal chemicals and desiccant dusts, are often ineffective and there is widespread resistance to acaricides across Europe. Alternative methods to control D. gallinae are urgently required and strategies include development of recombinant subunit vaccines and discovery of new potential acaricides. These strategies will benefit hugely from knowledge of the extent and rates of occurrence of genetic diversity within $D$. gallinae populations. In this study, genetic diversity of mites harvested from the UK and from sites across mainland Europe was studied at inter- and intra-farm levels. To achieve this, the genome analysis toolkit (GATK) best practices pipeline for single nucleotide polymorphism (SNP) and insertion/deletion variant calling was modified to be self-validating and used to identify 32,599 D. gallinae SNPs by comparing transcriptomic sequences (derived from mites harvested in Germany, Schicht et al.) with a $D$. gallinae genome assembly (derived from mites harvested in Scotland, Burgess et al.). Dermanyssus gallinae populations were sampled from 22 UK farms and 57 farms from 15 countries in mainland Europe. Analysis of 144 high-quality SNP markers across 117 pooled D. gallinae samples showed high spatial genetic diversity with significant linkage disequilibrium. Revisiting a subset of farms revealed notable temporal changes in genetic diversity.

\section{Introduction}

Dermanyssus gallinae (de Geer) is an obligatory blood feeding ectoparasite (Chauve, 1998). Primarily a parasite of birds, most notably laying hens, $D$. gallinae demonstrates considerable plasticity regarding host specificity and is capable of feeding on mammals, including humans (Valiente Moro et al., 2009). Dermanyssus gallinae is reported to have a worldwide distribution with high percentages of laying hen farms affected in European countries including Denmark, France, Romania, Italy, the Netherlands, Poland, Serbia and the United Kingdom (UK) (Sparagano et al., 2014, Hoglund et al., 1995, Guy et al., 2004, Fiddes et al., 2005, Cencek, 2003). In the UK, for example, between $60 \%$ and $85 \%$ of commercial hen egg laying systems are reported to be infested (Guy et al., 2004, Fiddes et al., 2005). Dermanyssus gallinae causes significant economic losses to the European poultry industry, estimated at €231 million per annum (Van Emous, 2017) attributed to higher feed conversion ratios, reduced quality and number of eggs, and the cost of control (Sparagano et al., 2014). Losses in the UK alone were estimated at €3 million in 2008 (Sparagano et al., 2014) and infestation rates have increased significantly since then. Affected hens become restless and display signs of itching/irritation with severe infections causing anaemia which can, especially in young hens at point of lay, cause death (Marangi et al., 2009b). Research by Kilpinen et al. (2005) on the influence of $D$. gallinae infestation on laying hen health showed a reduction in weight gain in young hens when compared to hens without infestation that persisted for at least 100 days (Kilpinen et al., 2005). In addition to the direct impacts of infestation, it is suggested that $D$. gallinae plays a role in bird-to-bird transmission of other pathogens including some that are zoonotic. For example, Newcastle disease virus has been isolated from D. gallinae mites (Arzey, 1990) and it has been shown experimentally that D. gallinae is capable of transmitting Pasteurella multocida and Salmonella enterica enterica serovar Gallinarum between birds (Petrov, 1975, Cocciolo et al., 2020).

Control of $D$. gallinae most commonly relies on the use of various classes of chemical compounds collectively referred to as 'acaricides' (Sparagano et al., 2014), although widespread resistance to many current products has been demonstrated (Marangi et al., 2012, Katsavou et al., 2020, Marangi et al., 2009a). Dermanyssus gallinae infestation is increasingly common in Europe, its presence enhanced by bans on the use of some effective chemical treatments, as well as legislative changes that has seen traditional caged systems replaced by enriched cages whose more complex structures facilitate the survival and spread of mites (Sparagano et al., 2009). Novel control methods are urgently required to reduce the health, welfare and economic impacts of $D$. gallinae and this includes screening for novel drugs as 
well as research into the development of recombinant vaccines. Vaccination appears to be a feasible approach for controlling D. gallinae (Bartley et al., 2012, Wright et al., 2016, Bartley et al., 2017, Bartley et al., 2015) but optimal antigens and strategies for delivery are yet to be determined. Given the rapidity with which $D$. gallinae populations can become resistant to many acaricides, it is not clear how mite populations would respond to being targeted by recombinant vaccines or the rapidity with which immune escape may occur. Improving knowledge of population structures for $D$. gallinae including the extent of naturally occurring genetic diversity and potential for transmission of resistance/escape alleles will provide data to underpin future models of genome evolution in response to novel control strategies.

The importance of $D$. gallinae and opportunities offered by modern sequencing and genotyping platforms has seen recent increases in genetic studies and data in public databases. Studies of diversity have mainly focused on the cytochrome c oxidase subunit I (COI) coding sequence (Marangi et al., 2009b, Oines and Brannstrom, 2011, Chu et al., 2015b, Roy and Buronfosse, 2011, Karp-Tatham et al., 2020), 16S rDNA (Roy et al., 2009b, Roy et al., 2010), and the internal transcribed spacer (ITS) regions (Marangi et al., 2012, Oines and Brannstrom, 2011, Chu et al., 2015a, Roy et al., 2010, Brannstrom et al., 2008, Potenza et al., 2009) and provide evidence for inter- and intra-national migration of mite populations. Studies by Roy et al. $(2009 ; 2010)$ on species specificity of the Dermanyssidae included several mite species and numerous isolates of $D$. gallinae from several European regions (Roy et al., 2009b, Roy et al., 2010). They reported intra-species variation rates of $<9 \%$ in the COI gene and, in conjunction with further analysis, suggested that $D$. gallinae represents a complex of hybridized lineages, possibly species, from a total of 35 haplotypes (Roy et al., 2010). Research by Roy et al. has previously revealed that $D$. gallinae sensu lato represents a species complex, with a minimum of at least two cryptic species present (where a cryptic species can be defined as one that cannot be distinguished just by morphological features). They define two cryptic species: $D$. gallinae sensu stricto and $D$. gallinae L1, with the species having been recorded in poultry farm populations across the world but not in other avians (Roy and Buronfosse, 2011, Roy et al., 2010, Roy et al., 2009a). In 2014, the first next-generation sequence (NGS) dataset for D. gallinae was transcriptome data published (Schicht et al., 2014). Total RNA was extracted from an acaricide-susceptible D. gallinae strain maintained at the University of Veterinary Medicine Hannover, Institute for Parasitology. Synthesis of cDNA was completed using a pool of RNA extracted from male and female mites, including all developmental stages in starved and fed states, followed by Roche 454 sequencing. The final dataset consisted of 267,464 transcribed sequences (231,657 singletons, 56 contigs and 35,751 isotigs) (Schicht et al., 2014). More recently, Burgess et al. released a draft genome assembly of the $D$. gallinae genome in 2018 (Burgess et al., 2018). They extracted genomic DNA from adult female $D$. gallinae mites and freshly laid eggs (collected from a layer farm in Scotland, UK), before using a combination of sequencings from PacBio and Oxford Nanopore Technologies MinION to produce a final assembly of 7,171 contigs with an assembled genome size of $959 \mathrm{Mb}$ (Burgess et al., 2018).

In this paper we used these published $D$. gallinae genomic and transcriptomic resources to identify a panel of highquality SNPs with utility for genome-wide population genetic analyses. Using a Mid-Plex SNP genotyping assay we have assessed the occurrence and extent of genetic diversity in UK and other European D. gallinae populations, defining the occurrence of spatial and temporal variation.

\section{Methods}

\subsection{Dermanyssus gallinae collection 2.1.1 United Kingdom}

Forty-one samples of Dermanyssus gallinae mites were collected from 22 farms across the UK between 2017 and 2020. The farms comprised 15 from England, three from Northern Ireland, three from Scotland and one from Wales (Figure 1, 
Supplementary Table S1). Mites were captured using cardboard traps as previously described (Nordenfors and Chirico, 2001). Thirty-one samples were collected from free range hen egg systems, of which 11 were organic, with seven collected from non-organic intensive enriched cage production systems. Three farms (three samples) provided $D$. gallinae mites but did not provide details relating to production system used or organic status. Nineteen farms were sampled once, contributing one sample from a single barn $(n=11)$, two samples from different sections of a single barn $(n=7)$, or two samples from different barns $(n=1)$. Three farms were revisited on three $(n=2)$ or four $(n=1)$ occasions, contributing multiple samples (Supplementary Table S1). Mites were either used directly (fresh), dried and frozen at $-20^{\circ} \mathrm{C}$, or preserved in ethanol (>70\% v/v; VWR International, Fontenary-sous-Bois, France). All farmers received instructions on how to carry out collection, a questionnaire and an information sheet relating to the project. Questions relating to production system and style, chicken husbandry and breed and farmer opinion regarding $D$. gallinae were included. Ethical approval was given by the Social Science Research Ethical Review Board at the Royal Veterinary College (URN SR2017-2018) for work pertaining to the questionnaire (supplied in Supplementary File 1). Confidential information was kept secure and all results were presented anonymously.

\subsubsection{Mainland Europe}

Dermanyssus gallinae collected from layer chicken farms in mainland Europe were received preserved in 70-100\% (v/v) ethanol, or alive in cardboard traps which were either used directly, dried and frozen at $-20^{\circ} \mathrm{C}$, or preserved in ethanol ( $>70 \%$ v/v; VWR International, Fontenary-sous-Bois, France). Samples were received from 15 countries from a total of 57 farms (Figure 1, Supplementary Table S2), with a mixture of samples collected and sent for this study and others sent from archives collected for previous research. Samples from farms were provided individually for all countries, except for Macedonia, where four farms were sampled and mites were pooled into a single tube.

\subsection{Geographical clustering}

Countries were grouped into four geographical clusters for analysis based on spatial proximity and climatic factors, with geographic cluster ID's assigned (Table 1). The UK formed one cluster, recognising its physical separation from mainland Europe.

Table 1

Geographical clustering of countries sampled for D. gallinae with assigned cluster number and countries included per country detailed

\begin{tabular}{|ll|}
\hline Geographical cluster & Countries included \\
\hline GC1 & United Kingdom \\
\hline GC2 & Belgium, Czech Republic, Denmark, Germany and the Netherlands \\
\hline GC3 & France, Portugal and Spain \\
\hline GC4 & Italy, Croatia, Slovenia \\
\hline GC5 & Albania, Greece, Romania, Macedonia \\
\hline
\end{tabular}

\subsection{DNA extraction and sample preparation}

Genomic DNA was extracted from 176 pooled D. gallinae populations using a Qiagen DNeasy blood and tissue kit (Qiagen $\mathrm{GmbH}$, Hilden, Germany) according to the manufacturer's protocol, with some modifications. Pools contained approximately 50-100 mites from all lifecycle stages and both sexes. Briefly, mites were homogenized by slicing the whole body with a sterile Agani $21 \mathrm{G} \times 1 \frac{1}{2}{ }^{\prime \prime}(0.8 \times 38 \mathrm{~mm})$ needle in a sterile $1.5 \mathrm{ml}$ Eppendorf tube. Proteinase $\mathrm{K}$ digestion was performed overnight at $56^{\circ} \mathrm{C}$. At the end of the protocol the resulting genomic DNA was eluted in $100 \mu \mathrm{l}$ 
DNase/RNase-free ultra-purified water (Invitrogen, Paisley, UK). Purified genomic DNA samples were stored at $-20^{\circ} \mathrm{C}$. All DNA samples were treated with RNase A (Thermo Fisher Scientific, Waltham, Massachusetts) to minimise RNA contamination. DNA concentration was analysed using a Qubit 4 Fluorometer (Thermo Fisher Scientific, Waltham, Massachusetts), as per the manufacturer's instructions.

\subsection{Sequence mapping, SNP calling, and annotation}

Individual paired-end read sets from the transcriptome produced by Schicht et al. (GenBank accession: GAIF01000000) were aligned to the reference draft D. gallinae genome assembly (Burgess et al., 2018) using BWA-MEM (BurrowsWheeler Aligner Maximal Exact Matcher) (Li, 2003) with the Simple Illumina mode for analysis and default parameters on the Galaxy platform (Afgan et al., 2018). Subsequently, the "MarkDuplicates" option in Picard tools (http://broadinstitute.github.io/picard/) was utilised to filter out potential PCR duplicates (Institute, 2020). SNP calling was undertaken following the Genome Analysis Toolkit (GATK) pipeline (McKenna et al., 2010). Due to a lack of preexisting known variants in D. gallinae, the GATK pipeline was modified for self-validation. Specifically, one initial round of SNP discovery was undertaken with no variant recalibration step completed and the resulting VCF file (produced through intersection of the VCF files from both individual paired-end read sets) was used in the second round of SNP discovery to enable variant recalibration. Base quality score was recalibrated to produce quality scores and corrected for variation in quality based on sequence context and machine cycle. Variant calling was performed using "UnifiedGenotyper" and "SelectVariants" arguments in GATK with the following filtering criteria to avoid possible false positives: SNPs with a Phred-scaled quality score of $<30$.

\subsection{SNP selection}

A panel of 100 high quality SNPs were identified based on a reductive system involving select criteria: minimum read depth of 36 and a minimum PHRED quality score of 350. SNPs were excluded if they were located within the first or last $50 \mathrm{bp}$ of a genomic contig. To optimise genome coverage SNPs were selected from individual contigs, with emphasis placed on individual SNPs incorporated from the largest contigs available in the genome assembly. An additional 57 SNPs were incorporated into the panel based on location within a region flanking one of the 100 targeted SNPs, given a minimum PHRED quality score of 250 and a minimum read depth of 20.

\subsection{SNP Genotyping and data processing 2.6.1 SNP Assay design}

Multiplex PCR Genotyping by sequencing was conducted by Eurofins Genomics using Mid-Plex genotyping method and analysed using Mid-Plex analysis software (Eurofins Genomics Europe Sequencing GmbH, Konstanz, Germany). For each individual candidate SNP, $150 \mathrm{bp}$ of sequence on each side of the target SNP was provided in 5'-3' orientation (301bp total) for customer primer design. Primers were designed by Eurofins and remain their proprietary property. Other known SNPs were coded using IUPAC codes. Assay optimisation was conducted by Eurofins Genomics with three different proprietary mixes tested, providing output relating to coverage class achieved. A total of $20 \mu$ l of extracted DNA was provided per sample. DNA concentration was measured using a NanoDrop 1000 spectrophotometer (NanoDrop Technologies, Wilmington, USA) with a range of 1.961 to $55.208 \mathrm{ng} / \mu \mathrm{l}$ (average $11.698 \mathrm{ng} / \mu \mathrm{l}$ ) or using a Qubit 4 Fluorometer (Thermo Fisher Scientific, Waltham, Massachusetts) with a range of 1.000-141.592 ng/ $\mu \mathrm{l}$ (average 22.261 $\mathrm{ng} / \mu \mathrm{l})$. The read out provided consisted of allele depth and genotype called. Initially, DNA samples extracted from 10-20 individual mites were pooled per farm for analysis (plate 1). Subsequently, DNA was extracted from pools of 30-50 mites to increase yield (plate 2).

\subsubsection{SNP analysis}


Three criteria were applied to raw SNP genotyping data to permit inclusion in analysis; 1 ) a minimum read cut off of three, 2) a SNP call rate of $70 \%$ or higher across the assay panel, and 3) SNPs called from $90 \%$ of samples or higher were retained for use. Heterozygous calls were converted to the dominant allele using raw allele read depths generated during sequencing. To achieve this, the allelic fraction was calculated based on ALT/(REF+ALT), where REF = reference allele read depth and $A L T=$ alternative allele read depth. A ratio of 0.6 or higher was used for stringent inference of alternative alleles and a ratio lower than 0.6 used to infer reference alleles.

\subsection{Genetic analysis}

SNP haplotype complexity was calculated for the entire dataset and biologically relevant subsets using DnaSP version 6.10.03 (Rozas et al., 2017). LIAN (Linkage Analysis) 3.0 was used to analyse haplotype linkage disequilibrium (LD) across D. gallinae populations (Haubold and Hudson, 2000). LIAN was used to calculate mean genetic diversity $(\mathrm{H})$, observed mismatch variance $\left(V_{D}\right)$, expected mismatch variance $(V e)$, standardized index of association (ISA), calculated significance $(P)$ and simulated $5 \%$ critical value $(L)$. Further linkage disequilibrium analysis was conducted using DnaSP version 6.10.03 (Rozas et al., 2017) to identify the number of SNP markers that demonstrated significant LD. The coefficient of LD (D) was normalised in DnaSP and presented as D' based upon the theoretical maximum difference between the observed and expected haplotype frequencies. $D^{\prime}$ is a standardisation method which is calculated by the comparison of $D$ compared to its maximum through the following equation $D^{\prime}=D / D$ max. The analysis was performed using a pairwise comparison for all polymorphic sites in the data, with statistical significance computed using the chisquare test with Bonferroni correction for multiple analyses applied. Phylogenetic comparison of sequences was achieved using Maximum Likelihood (ML) with the model selected based upon the highest Bayesian Information Criterion (BIC) using MEGA-X (Kumar et al., 2018). Statistical comparison was achieved using 1,000 bootstrap iterations. Network 10.0.0.0 (www.fluxus-engineering.com) was used to construct Median-Joining (MJ) networks (Bandelt et al., 1999). Dermanyssus gallinae samples with identical SNP sequences were designated as single, conserved haplotypes.

\section{Results}

One-hundred-and-seventeen pooled D. gallinae samples and 144 SNPs passed quality control and were analysed from 22 UK farms (41 samples) and 57 mainland European farms from 15 countries (76 samples). Questionnaire data was received from 18/22 UK farms. Time point replicates were included for three British farms (UK6, UK7 and UK11).

\subsection{Mapping, SNP calling, and annotation}

BWA-MEM mapping of transcriptomic data (Schicht et al., 2014) to the draft D. gallinae genome assembly (32) mapped $96.43 \%$ and $96.42 \%$ of reads from paired transcriptome datasets 1 and 2 , respectively. After the preliminary round of SNP discovery 62,592 and 69,440 SNPs were identified using each read set. Intersection analysis revealed 32,201 SNPs shared across both read sets, which were used for variant recalibration. A total of 65,428 SNPs (read set one) and 68,294 SNPs (read set two) were identified after variant recalibration, with 32,599 SNPs identified to be shared through VCF intersection. A total of 30,199 SNPs (62\%) were represented by REF and ALT alleles, whilst 12,480 (38\%) demonstrated only ALT alleles.

\subsection{Summary statistics from multiplex PCR SNP genotyping}

One-hundred-and-fifty-seven SNPs were analysed in two batches of 68 and 108 pooled D. gallinae samples (in plates 1 and 2 ), achieving $53.0 \%$ and $76.6 \%$ call rates, respectively (Tables $2-3$ ). The SNP call rate was variable across plates, but in total $80.0 \%$ and $97.5 \%$ of SNPs achieved a call rate of greater than $50 \%$ per plate (Table 2 ). SNP call rate was notably higher from plate 2 , where $47.2 \%$ of samples were characterised at more than $80 \%$ of SNPs. Comparison of results by sample over both plates found that $89 \%$ achieved a call rate of $50 \%$ or greater (Table 3 ). After quality control a total of 
117 samples with sufficient genotype coverage were retained for analysis, permitting 144 SNPs to be assessed (Table 3). Overall, 131 of these SNP markers were found to be polymorphic. Thirteen markers were invariant, 12 of which represented reference alleles and one represented the alternative from a total of 117 pooled $D$. gallinae samples.

Table 2

Summary SNP statistics of SNP genotyping data for two plates encompassing 108 and 68 samples, respectively. Details including overall cate rate and samples that passed quality control (QC)

\begin{tabular}{|lll|}
\hline & Plate 2 & Plate 1 \\
\hline Number of genotyped SNPs: & 157 & 157 \\
\hline Overall calling rate: & $53 \%$ & $76.6 \%$ \\
\hline Number of SNPs with $>80 \%$ call rate & $0(\%)$ & $74(47.2 \%)$ \\
\hline Number of SNPs between $\geq 50 \%$ and $<80 \%$ call rate & $126(80.0 \%)$ & $79(50.3 \%)$ \\
\hline Number of SNPs between $>10 \%$ and $<50 \%$ call rate & $31(20.0 \%)$ & $4(2.5 \%)$ \\
\hline Number of SNPs with $<10 \%$ call rate & $0(0.0 \%)$ & $0(0.0 \%)$ \\
\hline
\end{tabular}

Table 3

Summary SNP statistics of SNP genotyping data for two plates encompassing 108 and 68 samples, respectively. Details including overall cate rate and samples that passed quality control (QC)

\begin{tabular}{|lll|}
\hline & Plate 2 & Plate 1 \\
\hline Number of analysed SAMPLES: & 68 & 108 \\
\hline Number of SAMPLES with $>90 \%$ call rate: & $31(46 \%)$ & $71(65.7 \%)$ \\
\hline Number of SAMPLES between $\geq 50 \%$ and $>90 \%$ call rate & $8(12 \%)$ & $17(15.7 \%)$ \\
\hline Number of SAMPLES between $>10 \%$ and $<50 \%$ call rate & $10(15 \%)$ & $6(5.5 \%)$ \\
\hline Number of SAMPLES with $<10 \%$ call rate & $18(26 \%)$ & $14(12.9 \%)$ \\
\hline Number of SAMPLES that passed QC: & 39 & 78 \\
\hline
\end{tabular}

\subsection{Nucleotide analysis}

Mean genetic diversity for all samples combined was 0.3478 (calculated using LIAN; Table 4). Based on geographical proximity, cluster two had the lowest diversity (0.2915; Belgium, Czech Republic, Denmark, Germany and the Netherlands) whilst cluster four had the highest (0.3581; Italy, Croatia and Slovenia). Across all populations, no shared haplotypes were observed with a unique haplotype recorded for every sample (Table 4). 
Table 4

Linkage analysis of 145 SNP markers for 117 pooled D. gallinae samples using LIAN (84). Results shown for each dataset analysed including the full dataset, and subsets representing different production systems across Europe, four geographic clusters, UK production system and UK organic status. The number of samples and number of haplotypes

included for each dataset was a shown. *Four farms were removed from production type analysis due to lack of information regarding production system. ** Total of 25 farms included in organic status analysis, as intensive farms sampled were not organic. $\mathrm{H}$ : mean genetic diversity, $\operatorname{Var}\left(\mathrm{V}_{\mathrm{D}}\right)$ : observed mismatch variance, $\mathrm{V}_{\mathrm{e}}$ : expected mismatch variance, $\mathrm{I}_{\mathrm{A}} \mathrm{S}$ : Standardized index of association, $\mathrm{P}$ : calculated significance, $\mathrm{L}$ : Simulated $5 \%$ critical value

\begin{tabular}{|c|c|c|c|c|c|c|c|c|c|}
\hline $\begin{array}{l}\text { Dataset } \\
\text { analysed }\end{array}$ & $\begin{array}{l}\text { No. of } \\
\text { samples }\end{array}$ & $\begin{array}{l}\text { No. of } \\
\text { haplotypes }\end{array}$ & $H$ & $\mathrm{~V}_{\mathrm{D}}$ & $\mathrm{V}_{\mathrm{e}}$ & $\mathrm{I}_{A}^{\mathrm{S}}$ & $\operatorname{Var}\left(V_{\mathrm{D}}\right)$ & $P$ & $L$ \\
\hline All & 117 & 117 & $\begin{array}{l}0.3478 \\
+/- \\
0.0142\end{array}$ & 159.9252 & 28.6566 & 0.0318 & 1.2191 & $<0.01$ & 30.5589 \\
\hline
\end{tabular}

\section{Production type*}

\begin{tabular}{|c|c|c|c|c|c|c|c|c|c|}
\hline $\begin{array}{l}\text { Free- } \\
\text { range/Rural } \\
\text { farm/Private } \\
\text { flock }\end{array}$ & 40 & 40 & $\begin{array}{l}0.3224 \\
+/- \\
0.0159\end{array}$ & 144.2106 & 26.3837 & 0.0310 & 3.7205 & $<0.01$ & 29.7289 \\
\hline $\begin{array}{l}\text { Intensive } \\
\text { systems }\end{array}$ & 72 & 72 & $\begin{array}{l}0.3544 \\
+/- \\
0.0140\end{array}$ & 161.5141 & 29.0589 & 0.0317 & 3.5772 & $<0.01$ & 32.9229 \\
\hline
\end{tabular}

\section{Geographic cluster (see Table 1)}

\begin{tabular}{|c|c|c|c|c|c|c|c|c|c|}
\hline GC1 (UK) & 41 & 41 & $\begin{array}{l}0.3079 \\
+/- \\
0.0155\end{array}$ & 150.3279 & 25.9079 & 0.0334 & 6.7851 & $<0.01$ & 30.6088 \\
\hline GC2 & 20 & 20 & $\begin{array}{l}0.2915 \\
+/- \\
0.0150\end{array}$ & 143.9070 & 25.2254 & 0.0327 & 12.4182 & $<0.01$ & 31.8181 \\
\hline GC3 & 24 & 24 & $\begin{array}{l}0.3087 \\
+/- \\
0.0159\end{array}$ & 128.9451 & 25.6848 & 0.0279 & 14.3548 & $<0.01$ & 32.1451 \\
\hline GC4 & 12 & 12 & $\begin{array}{l}0.3581 \\
+/- \\
0.0195\end{array}$ & 64.8096 & 25.4300 & 0.0108 & 25.6823 & $<0.01$ & 34.7173 \\
\hline GC5 & 20 & 20 & $\begin{array}{l}0.3121 \\
+/- \\
0.0159\end{array}$ & 289.5484 & 25.8515 & 0.0708 & 13.0487 & $<0.01$ & 32.8403 \\
\hline
\end{tabular}

\section{Production system (UK)*}

\begin{tabular}{|llllllllll} 
Intensive & 7 & 7 & $\begin{array}{l}0.3458 \\
+/-\end{array}$ & 217.1286 & 25.2472 & 0.0528 & 98.5553 & $<0.01$ & 46.4286 \\
& 0.0190 & & & & & & \\
Free-range & 31 & 31 & $\begin{array}{l}0.2997 \\
+/-\end{array}$ & 126.8595 & 25.1463 & 0.0281 & 7.7630 & $<0.01$ & 29.8060 \\
& & & & & & & & &
\end{tabular}

\section{Organic status (UK) **}

$\begin{array}{llllllllll}\text { Organic } & 11 & 11 & \begin{array}{l}0.2872 \\ +/-\end{array} & 169.6889 & 22.4079 & 0.0456 & 41.3236 & <0.01 & 36.2343 \\ & & & & & & & & \end{array}$




\begin{tabular}{|llllllllll|}
\hline $\begin{array}{l}\text { Dataset } \\
\text { analysed }\end{array}$ & $\begin{array}{l}\text { No. of } \\
\text { samples }\end{array}$ & $\begin{array}{l}\text { No. of } \\
\text { haplotypes }\end{array}$ & $H$ & $V_{D}$ & $V_{e}$ & $I_{A} s^{2}$ & $\operatorname{Var}\left(V_{D}\right)$ & $P$ & $L$ \\
\hline Non-organic & 27 & 27 & $\begin{array}{l}0.2985 \\
+/- \\
0.0156\end{array}$ & 139.0519 & 25.2702 & 0.0313 & 12.1642 & $<0.01$ & 32.6074 \\
\hline
\end{tabular}

\subsubsection{Production systems}

Comparing production systems across Europe found no statistically significant difference in mean genetic diversity, with intensive production systems marginally higher. It should be noted that the UK accounted for most of the free-range farms sampled (77.5\%) and conversely samples from UK intensive farms represented just 7/ 41 pools analysed and should be open to interpretation. In the UK, lower genetic diversity was observed in organic free-range samples (Table 4).

\subsection{Linkage disequilibrium}

Linkage analysis utilising LIAN revealed significant linkage disequilibrium in the full dataset, all geographic clusters analysed, and all production systems (Table 4). Linkage disequilibrium (LD) analysis completed using DnaSP included 1653 pairwise comparisons and revealed a total of 483 significant pairwise comparisons by chi-square test, 74 of which remained significant using the Bonferroni procedure (average D' $=0.42927$ ). D' (normalised D) was positive at all statistically significant sites, with and without the Bonferroni correction, indicating that these markers occurred together in the same haplotype more than expected.

\subsection{Phylogenetic analysis and Network analysis}

Exactly 117 haplotypes were identified through network analysis, corresponding to the 117 D. gallinae pooled samples analysed after quality control, with no shared haplotypes between samples (Figure 2). Clustering of samples originating from the same country was observed for countries such as Belgium and Romania (Figure 2). Conversely, haplotypes from more intensively sampled countries such as the UK were distributed across the network map, sharing phylogenetic relatedness with multiple countries. ML phylogenetic analysis revealed two main clades $(A$ and $B)$, with four subgroups ( $A a, A b, B a$ and $B b ;$ Figure 3). Samples from the UK were predominantly found in clade B with 39/41 samples in subgroups $\mathrm{Ba}$ and $\mathrm{Bb}$ (Figure 2). Clade A contained 61 samples (47 in Aa and 14 in Ab) and clade B contained 56 samples (14 in $\mathrm{Ba}$ and 42 in $\mathrm{Bb}$ ). Cluster Aa was most geographically diverse, with samples from 13 countries present, whilst Clade Ba contained samples from just four countries (Figure 3). Clustering of samples from Romania and Belgium suggest less differentiation between $D$. gallinae populations in these countries when compared to Portugal and the UK, where haplotypes were spread across the network map with close phylogeny to multiple countries. Farms sampled in Romania and Belgium were from a limited geographic range, especially when compared to the size of the country in the case of Romania (Figure 1).

\subsubsection{United Kingdom}

From the UK, network analysis of 41 pooled $D$. gallinae samples from 22 UK farms demonstrated 41 individual haplotypes with spatial structuring. Analysis based on nation of origin (England, Northern Ireland, Scotland and Wales) revealed close phylogenetic relationships between all countries with no separation on the network map of individual countries (Figure 4A). Samples from UK farms were shown to cluster together (27/41 farms), in close relationship with farms from Albania, Greece and the Netherlands. In contrast, some individual haplotypes from UK were shown to be phylogenetically related to those from farms in Belgium, Portugal and Romania (Figure 2). Analysis of variation by production system in the UK demonstrated close phylogenetic relationships between all production systems and no clear differentiation between organic or non-organic free-range farms (Figure 4B). 
Haplotypes were assessed from samples collected from the same barn on farm UK6 on four occasions, starting with a single collection from one flock (time 0 ), followed by another three samples 14, 15 and 20 months later from a subsequent flock. While all four haplotypes were similar, those collected from the same flock were most closely related (Figure 4A). A close phylogenetic relationship was observed between three samples collected from the same barn close together in time (six months apart; 14, 15 and 20 months after initial sampling, respectively), with greater phylogenetic separation seen from the initial (more distant) time of sampling (Figure 4B). The flock was 1.3 years old at the first time point $(+0$, Figure $4 B)$, with a change in flock before the subsequent time points $(+14,+15,+20)$, providing one plausible explanation for phylogenetic differentiation. Samples collected from two other barns on farm UK6 were phylogenetically distinct, with haplotypes from barns $b$ and c more closely related to UK7 and UK18, respectively

Samples from UK7 were found distributed across the network map, whilst samples from UK11 clustered close together. Two barns sampled from UK14 also demonstrated distinct haplotypes with clear phylogenetic differentiation between populations, with the haplotype from one barn in haplogroup $A$ and the haplotype from the other barn found in haplogroup B (Figure 4B).

\section{Discussion}

Knowledge of relevant population structure and pre-existing genetic diversity will be valuable for accurate prediction of longevity and efficacy of novel control methods against $D$. gallinae. To gain insights into $D$. gallinae population genetics, a custom Mid-Plex SNP genotyping assay was developed. 157 SNP markers were chosen from a panel of 30,199 high quality SNPs identified within the $D$. gallinae genome by comparison with transcriptomic data using an adapted selfvalidating GATK pipeline. A total of 144 SNP markers were analysed after three criteria were applied to raw SNP genotyping data, with 132 SNPs being informative (i.e. polymorphic). A distinct population structure was observed including high genetic diversity, defined by multiple unique haplotypes, together with notable linkage disequilibrium.

No statistical difference in genetic diversity of $D$. gallinae populations from differing production systems was noted although non-organic farms in the UK had a consistent tendency to greater genetic diversity than organic farms. This could be due to strict limitations on use of chemicals in organic farms (Zenner et al., 2003) as Roy and Buronfosse have shown that selection imposed by control measures and hygiene practices affect allelic composition of $D$. gallinae populations (Roy and Buronfosse, 2011). Thus, greater pressure for diversifying selection exerted by the inclusion of chemical treatments alongside other methods may underpin increased genetic diversity in non-organic farms. Typically, selection pressure from the use of highly effective chemical control would be expected to reduce genetic diversity through imposition of a bottleneck (Coles and Dryden, 2014). However, personal observations during mite collection in the UK as well as questionnaire data revealed that for many farms a broad range of control measures were adopted, including chemical control, desiccant dusts, natural control measures and hygiene related measures (Supplementary Table S3) (Guy et al., 2004). This could result in diversifying selection (i.e. little or no directional selection on specific haplotypes) across the UK as populations of $D$. gallinae at each farm are under locally specific selection pressures related to the combination of control measure(s) in place.

Results from phylogenetic, network and linkage analyses suggest a spatial structuring of genetic diversity in $D$. gallinae across Europe, with high haplotype numbers for populations and significant linkage disequilibrium in all populations $(P<0.01)$ (Table 4). Admixture within UK farms (i.e. between Scotland, Ireland, Wales and England) was observed through close phylogeny and there were also close phylogenetic relationships between mites sampled from farms in different European countries. This is indicative of on-going admixture between farms in a single country and between countries, which has also been shown in studies of COI genetic diversity (Oines and Brannstrom, 2011, Chu et al., 2015a, KarpTatham et al., 2020, Roy et al., 2021). Spatial structuring of genetic diversity can occur when gene flow is insufficient for homogenisation of allele frequencies throughout a studied area (Bohonak, 1999). As well as gene flow and dispersal, 
genetic structure can be influenced by genetic drift in small populations, which impacts genetic structure through increased differentiation (Levy and Neal, 1999). The sampling strategy for this study predominantly focused on commercial laying farms, where it is likely that the $D$. gallinae populations sampled repeatedly started from small founding populations including survivors from farm cleaning and/or new mites bought onto farms via infested hens or equipment (Roy et al., 2021). A subdivision in populations or changes in population size, as well as the exchange of individuals across populations, can all affect LD (Slatkin, 1975, Slatkin, 2008). Intentional or unintentional mixing of individuals from subpopulations with differing allele frequencies creates genomic LD (Mitton et al., 1973, Nei and Li, 1973). Mixing of $D$. gallinae populations during contraction and expansion can occur when infested transport/equipment or hens are bought onto farms and this can occur within individual countries from suppliers or across countries through trade links. This is supported by previous research focusing on Tropomyosin. Roy and Buronfosse demonstrated marked divergence between tropomyosin alleles carried by $D$. gallinae mites from farms compared to wild avifauna (Roy and Buronfosse, 2011). From poultry farms, two to three very distant tropomyosin alleles were commonly observed with heterozygous status, whilst in wild avifauna heterozygotes carried less distant alleles that could typically be assigned to one of three groups found in poultry farms (Roy and Buronfosse, 2011). The authors hypothesised that the major divergence of alleles observed in D. gallinae collected from farms could result from hybridisation events caused through the breakdown of geographical barriers by long-distance transfer through human involvement.

Network analysis revealed 117 unique haplotypes across all $D$. gallinae populations sampled with no shared haplotypes (Figure 2). As genetic drift can impact genetic structure in small populations through increased differentiation (Levy and Neal, 1999), it is possible that small founding populations in farms have differentiated through genetic drift during population expansion and colonisation of individual farms, shifting allelic frequencies to form individual haplotypes. Investigation into COI diversity in Sweden and Norway revealed signs of differentiation between populations, consistent with the varied administrative processes in each country (Oines and Brannstrom, 2011). While the haplotypes detected were closely related, none were shared between the countries (Oines and Brannstrom, 2011). Sweden was also shown to share haplotypes with other European populations, possibly due to foreign trade and EU status (Oines and Brannstrom, 2011). Results reported here are consistent with analyses based upon fragments of the COI gene. Roy et al. demonstrated that $D$. gallinae is structured into three distinct mitochondrial groups (haplogroups A, B and C) (Oh et al., 2019), adding to results from previously published studies (Oh et al., 2019, Ciloglu et al., 2020, Oines and Brannstrom, 2011, Chu et al., 2015a, Roy and Buronfosse, 2011). Network analysis conducted by Roy et al., revealed recurrent signs of expansion when several star patterns were formed, indicative of rare variants (one to two mutations) very closely related to a dominant haplotype consistent with the population expansion/contraction described above (Roy et al., 2021). These three haplogroups have also been widely recorded worldwide, including Australia (Roy and Buronfosse, 2011), Brazil (Roy and Buronfosse, 2011), Europe (Oines and Brannstrom, 2011), Japan (Chu et al., 2015a) and Korea (Oh et al., 2019). This distribution is likely entirely the result of human movement and activity.

Distinct haplotypes were observed from all samples when farms were visited on multiple occasions (UK6, UK7 and UK11), indicating regular changes in D. gallinae populations over time. Different patterns of phylogeny were observed for each farm, implying that changes in diversity were also related to individual farms and do not follow a universal pattern. Distinct populations could possibly be the result of effective control measures and high hygiene measures preventing unintentional mixing of populations, producing three separate subpopulations residing on the same farm. Individuality in temporal changes to genetic diversity in farms might be related to differences in management factors including the choice of control measure(s) against $D$. gallinae, production and housing system, hygiene practices and staff compliance, and flock size. Closely comparable haplotypes detected in the same barn (UK6) from close time points could reflect different subpopulations emerging during expansion in the new flock. In contrast, samples collected from UK7 just two months apart demonstrated clear phylogenetic separation, possibly representing two or more 
subpopulations of $D$. gallinae in the same flock. Samples collected from different areas of the same barn (UK6) at the same time point also reflected individual haplotypes. This type of variation was also observed in mites collected from Romanian farms (ROM1-ROM6), whereby two biological replicates were sampled from different parts of each farm and all represented unique haplotypes. Under optimal conditions, $D$. gallinae can complete its lifecycle within 7-10 days (Maurer and Baumgartner, 1992), so population expansion can occur very quickly, with rapid shifts in allelic frequencies theoretically possible. These results from individual barns imply that an integrated pest management (IPM) control against $D$. gallinae could work effectively to help maintain efficacy of future control methods. Results from UK6, UK7 and UK 11 show clear indications of changes in $D$. gallinae over the course of time, however a greater number of $D$. gallinae pools from each time point, a greater number of time points and increased number of farms sampled over time would be required to provide a deeper understanding in the changes in $D$. gallinae populations. These pilot results focusing on temporal changes indicate a definitive shift in the genetic diversity of $D$. gallinae populations over time and the presence of potential subpopulations within individual barns. The number of samples required to capture the true extent of diversity within even a single barn is not clear. In terms of control measures, this indicates a high capacity for adaptation by $D$. gallinae with rapid shifts in allelic frequencies. This further highlights the requirement for novel chemical and vaccine controls to target highly conserved genes with essential functions that are less likely to evolve towards chemical resistance or vaccine escape. An integrated chemical/vaccine/environmental control strategy is most likely to ensure long term sustainable control of $D$. gallinae. Integration of novel control methods (e.g. a vaccine or new chemicals) that are targeted at highly conserved genes could be effectively used on each barn as an individual population with minimal cross over. Testing for acaricide resistance using genetic markers as seen in Katsavou et al., (16) could be included in an IPM strategy, with the spread of resistant mites reduced through segregation of populations from individual barns.

Results demonstrated here regarding the genetic diversity of $D$. gallinae have implications for future control measures and could directly contribute to targeting novel control measures at highly conserved genes that encode proteins with an essential function. These genes are less likely to evolve diversity that promotes chemical resistance or vaccine escape due to selection pressure to maintain sequence conservation, making them optimal drug and vaccine candidates (Olsen et al., 2015, Duffield et al., 2010).

\section{Conclusion}

Analysis of 144 SNP markers from 117 pooled D. gallinae samples collected from across the UK and mainland Europe showed high spatial genetic diversity, with no conserved haplotypes detected at more than one location or on more than one occasion. Significant linkage disequilibrium was detected across all populations indicating historical and on-going admixture between $D$. gallinae populations.

\section{Declarations}

\section{Ethical Approval and Consent to Participate}

Ethical approval was given by the Social Science Research Ethical Review Board at the Royal Veterinary College (URN SR2017-2018) for work pertaining to the questionnaire (supplied in Supplementary File 1). Confidential information was kept secure and all results were presented anonymously.

\section{Competing Interests}

The authors declare that the research was conducted in the absence of any commercial or financial relationships that could be construed as a potential conflict of interest.

\section{Consent for Publication}

Page 12/20 
Not applicable.

\section{Authors' contributions}

EKT and DB were involved in molecular biology work and preparation of samples. FT and DB secured funding through the BEMB for this work to be undertaken. EKT, TM, FT, DB and AN contributed to sample collection. EKT, DX and DB completed genetic analysis. AN provided access to materials required for completion of the work. All authors contributed to writing and editing of the publication.

\section{Acknowledgments}

This study was funded by the British Egg Marketing Board Trust (BEMB) as part of a four year PhD studentship at the Royal Veterinary College. The funders had no direct role in the design of the study, analysis, publishing decision or preparation of the manuscript. We wish to thank all UK farmers for taking the time to set cardboard traps and provide $D$. gallinae samples. Extended thanks to the following for contribution of European samples: Athanasious Angelou, Veli YilgÇr ,sirak, Monique Mul, Cristian Magdas, Susanne Kabell, Lise Roy, Miroslav Radeski, Tana Shtylla Kika, Antonio Camarda, Danijela Horvatek Tomic, Martina Lichovnikova and José Francisco Lima Barbero. We also wish to thank Fernando Moreira and Rui Fortunato. The author of the research unit CECAV received funding from the Fundação para a Ciência e Tecnologia (FCT), reference of the project UIDB/CVT/0772/2020.

\section{Availability of data and materials}

The datasets during and/or analysed during the current study available from the corresponding author on reasonable request.

\section{References}

1. AFGAN, E., BAKER, D., BATUT, B., VAN DEN BEEK, M., BOUVIER, D., CECH, M., CHILTON, J., CLEMENTS, D., CORAOR, N., GRÜNING, B. A., GUERLER, A., HILLMAN-JACKSON, J., HILTEMANN, S., JALILI, V., RASCHE, H., SORANZO, N., GOECKS, J., TAYLOR, J., NEKRUTENKO, A. \& BLANKENBERG, D. 2018. The Galaxy platform for accessible, reproducible and collaborative biomedical analyses: 2018 update. Nucleic Acids Res, 46, W537-W544.

2. ARZEY, G. 1990. Mechanism of spread of Newcastle disease. New South Wales Agriculture and Fisheries Bulletin, $41,12$.

3. BANDELT, H. J., FORSTER, P. \& RÖHL, A. 1999. Median-joining networks for inferring intraspecific phylogenies. Mol Biol Evol, 16, 37-48.

4. BARTLEY, K., HUNTLEY, J. F., WRIGHT, H. W., NATH, M. \& NISBET, A. J. 2012. Assessment of cathepsin D and L-like proteinases of poultry red mite, Dermanyssus gallinae (De Geer), as potential vaccine antigens. Parasitology, 139, 755-65.

5. BARTLEY, K., TURNBULL, F., WRIGHT, H. W., HUNTLEY, J. F., PALAREA-ALBALADEJO, J., NATH, M. \& NISBET, A. J. 2017. Field evaluation of poultry red mite (Dermanyssus gallinae) native and recombinant prototype vaccines. Veterinary Parasitology, 244, 25-34.

6. BARTLEY, K., WRIGHT, H. W., HUNTLEY, J. F., MANSON, E. D., INGLIS, N. F., MCLEAN, K., NATH, M., BARTLEY, Y. \& NISBET, A. J. 2015. Identification and evaluation of vaccine candidate antigens from the poultry red mite (Dermanyssus gallinae). Int J Parasitol, 45, 819-30.

7. BOHONAK, A. J. 1999. Dispersal, gene flow, and population structure. Q Rev Biol, 74, 21-45.

8. BRANNSTROM, S., MORRISON, D. A., MATTSSON, J. G. \& CHIRICO, J. 2008. Genetic differences in internal transcribed spacer 1 between Dermanyssus gallinae from wild birds and domestic chickens. Med Vet Entomol, 22, 
152-5.

9. BURGESS, S. T. G., BARTLEY, K., NUNN, F., WRIGHT, H. W., HUGHES, M., GEMMELL, M., HALDENBY, S., PATERSON, S., ROMBAUTS, S., TOMLEY, F. M., BLAKE, D. P., PRITCHARD, J., SCHICHT, S., STRUBE, C., OINES, O., VAN LEEUWEN, T., VAN DE PEER, Y. \& NISBET, A. J. 2018. Draft Genome Assembly of the Poultry Red Mite, Dermanyssus gallinae. Microbiol Resour Announc, 7.

10. CENCEK, T. 2003. Prevalence of Dermanyssus gallinae in poultry farms in silesia region in Poland. Bulletin of the Veterinary Institute in Pulawy, 47, 465-469.

11. CHAUVE, C. 1998. The poultry red mite Dermanyssus gallinae (De Geer, 1778): current situation and future prospects for control. Vet Parasitol, 79, 239-45.

12. CHU, T. T., MURANO, T., UNO, Y., USUI, T. \& YAMAGUCHI, T. 2015a. Molecular epidemiological characterization of poultry red mite, Dermanyssus gallinae, in Japan. J Vet Med Sci, 77, 1397-403.

13. CHU, T. T. H., MURANO, T., UNO, Y., USUI, T. \& YAMAGUCHI, T. 2015b. Molecular epidemiological characterization of poultry red mite, Dermanyssus gallinae, in Japan. Journal of Veterinary Medical Science, 77, 1397-1403.

14. CILOGLU, A., YILDIRIM, A., ONDER, Z., YETISMIS, G., DUZLU, O., SIMSEK, E. \& INCI, A. 2020. Molecular characterization of poultry red mite, Dermanyssus gallinae lineages in Turkey and first report of Plasmodium species in the mite populations. International Journal of Acarology, 46, 241-246.

15. COCCIOLO, G., CIRCELLA, E., PUGLIESE, N., LUPINI, C., MESCOLINI, G., CATELLI, E., BORCHERT-STUHLTRÄGER, M., ZOLLER, H., THOMAS, E. \& CAMARDA, A. 2020. Evidence of vector borne transmission of Salmonella enterica enterica serovar Gallinarum and fowl typhoid disease mediated by the poultry red mite, Dermanyssus gallinae (De Geer, 1778). Parasit Vectors, 13, 513.

16. COLES, T. B. \& DRYDEN, M. W. 2014. Insecticide/acaricide resistance in fleas and ticks infesting dogs and cats. Parasit Vectors, 7, 8.

17. DUFFIELD, M., COOPER, I., MCALISTER, E., BAYLISS, M., FORD, D. \& OYSTON, P. 2010. Predicting conserved essential genes in bacteria: in silico identification of putative drug targets. Mol Biosyst, 6, 2482-9.

18. FIDDES, M. D., LE GRESLEY, S., PARSONS, D. G., EPE, C., COLES, G. C. \& STAFFORD, K. A. 2005. Prevalence of the poultry red mite (Dermanyssus gallinae) in England. Vet Rec, 157, 233-5.

19. GUY, J. H., KHAJAVI, M., HLALEL, M. M. \& SPARAGANO, O. 2004. Red mite (Dermanyssus gallinae) prevalence in laying units in Northern England. Br Poult Sci, 45 Suppl 1, S15-6.

20. HAUBOLD, B. \& HUDSON, R. R. 2000. LIAN 3.0: detecting linkage disequilibrium in multilocus data. Linkage Analysis. Bioinformatics, 16, 847-8.

21. HOGLUND, J., NORDENFORS, H. \& UGGLA, A. 1995. Prevalence of the poultry red mite, Dermanyssus gallinae, in different types of production systems for egg layers in Sweden. Poult Sci, 74, 1793-8.

22. INSTITUTE, B. 2020. Picard [Online]. Available: http://broadinstitute.github.io/picard/ [Accessed 27/04/2020 2020].

23. KARP-TATHAM, E., KÜSTER, T., ANGELOU, A., PAPADOPOULOS, E., NISBET, A. J., XIA, D., TOMLEY, F. M. \& BLAKE, D. P. 2020. Phylogenetic Inference Using Cytochrome C Oxidase Subunit I (COI) in the Poultry Red Mite,. Front Vet Sci, 7, 553.

24. KATSAVOU, E., VLOGIANNITIS, S., KARP-TATHAM, E., BLAKE, D. P., ILIAS, A., STRUBE, C., KIOULOS, I., DERMAUW, W., VAN LEEUWEN, T. \& VONTAS, J. 2020. Identification and geographical distribution of pyrethroid resistance mutations in the poultry red mite Dermanyssus gallinae. Pest Manag Sci, 76, 125-133.

25. KILPINEN, O., ROEPSTORFF, A., PERMIN, A., NORGAARD-NIELSEN, G., LAWSON, L. G. \& SIMONSEN, H. B. 2005. Influence of Dermanyssus gallinae and Ascaridia galli infections on behaviour and health of laying hens (Gallus gallus domesticus). Br Poult Sci, 46, 26-34. 
26. KUMAR, S., STECHER, G., LI, M., KNYAZ, C. \& TAMURA, K. 2018. MEGA X: Molecular Evolutionary Genetics Analysis across Computing Platforms. Mol Biol Evol, 35, 1547-1549.

27. LEVY, F. \& NEAL, C. L. 1999. Spatial and temporal genetic structure in chloroplast and allozyme markers in phacelia dubia implicate genetic drift. Heredity (Edinb), 82 Pt 4, 422-31.

28. LI, H. 2003. Aligning sequence reads, clone sequences and assembly contigs with BWA-MEM. ArXiv, 1.

29. MARANGI, M., CAFIERO, M. A., CAPELLI, G., CAMARDA, A., SPARAGANO, O. A. \& GIANGASPERO, A. 2009a. Evaluation of the poultry red mite, Dermanyssus gallinae (Acari: Dermanyssidae) susceptibility to some acaricides in field populations from Italy. Exp Appl Acarol, 48, 11-8.

30. MARANGI, M., DE LUNA, C. J., CAFIERO, M. A., CAMARDA, A., LE BOUQUIN, S., HUONNIC, D., GIANGASPERO, A. \& SPARAGANO, O. A. 2009b. Phylogenetic relationship between Dermanyssus gallinae populations in European countries based on mitochondrial COI gene sequences. Exp Appl Acarol, 48, 143-55.

31. MARANGI, M., MORELLI, V., PATI, S., CAMARDA, A., CAFIERO, M. A. \& GIANGASPERO, A. 2012. Acaricide residues in laying hens naturally infested by red mite Dermanyssus gallinae. PLoS One, 7, e31795.

32. MAURER, V. \& BAUMGARTNER, J. 1992. Temperature influence on life table statistics of the chicken mite Dermanyssus gallinae (Acari: Dermanyssidae). Exp Appl Acarol, 15, 27-40.

33. MCKENNA, A., HANNA, M., BANKS, E., SIVACHENKO, A., CIBULSKIS, K., KERNYTSKY, A., GARIMELLA, K., ALTSHULER, D., GABRIEL, S., DALY, M. \& DEPRISTO, M. A. 2010. The Genome Analysis Toolkit: A MapReduce framework for analyzing next-generation DNA sequencing data. Genome Research, 20, 1297-1303.

34. MITTON, J. B., KOEHN, R. K. \& PROUT, T. 1973. Population genetics of marine pelecypods. III. Epistasis between functionally related isoenzymes of Mytilus edulis. Genetics, 73, 487-496.

35. NEI, M. \& LI, W. H. 1973. Linkage disequilibrium in subdivided populations. Genetics, 75, 213-9.

36. NORDENFORS, H. \& CHIRICO, J. 2001. Evaluation of a sampling trap for Dermanyssus gallinae (Acari: Dermanyssidae). J Econ Entomol, 94, 1617-21.

37. OH, S., NOH, G., YI, S., DO, Y., KIM, E. \& YOO, J. 2019. Molecular epidemiological characterization of poultry red mite (Dermanyssus gallinae) collected from Korea. Korean Journal of Veterinary Service, 42, 161-167.

38. OINES, O. \& BRANNSTROM, S. 2011. Molecular investigations of cytochrome c oxidase subunit I (COI) and the internal transcribed spacer (ITS) in the poultry red mite, Dermanyssus gallinae, in northern Europe and implications for its transmission between laying poultry farms. Med Vet Entomol, 25, 402-12.

39. OLSEN, L. R., SIMON, C., KUDAHL, U. J., BAGGER, F. O., WINTHER, O., REINHERZ, E. L., ZHANG, G. L. \& BRUSIC, V. 2015. A computational method for identification of vaccine targets from protein regions of conserved human leukocyte antigen binding. BMC Med Genomics, 8 Suppl 4, S1.

40. PETROV, D. 1975. Study of Dermanyssus gallinae as a carrier of Pasteurella multocida. Vet Med Nauki, 12, 32-36.

41. POTENZA, L., CAFIERO, M. A., CAMARDA, A., LA SALANDRA, G., CUCCHIARINI, L. \& DACHA, M. 2009.

Characterization of Dermanyssus gallinae (Acarina: Dermanissydae) by sequence analysis of the ribosomal internal transcribed spacer regions. Vet Res Commun, 33, 611-8.

42. ROY, L. \& BURONFOSSE, T. 2011. Using mitochondrial and nuclear sequence data for disentangling population structure in complex pest species: a case study with Dermanyssus gallinae. PLoS One, 6, e22305.

43. ROY, L., DOWLING, A., CHAUVE, C. M. \& BURONFOSSE, T. 2009a. Delimiting species boundaries within Dermanyssus Dugès, 1834 (Acari: Mesostigmata) using a total evidence approach. Molecular Phylogenetics and Evolution, 50, 446-470.

44. ROY, L., DOWLING, A. P., CHAUVE, C. M. \& BURONFOSSE, T. 2010. Diversity of phylogenetic information according to the locus and the taxonomic level: an example from a parasitic mesostigmatid mite genus. Int J Mol Sci, 11, 170434.

Page $15 / 20$ 
45. ROY, L., DOWLING, A. P., CHAUVE, C. M., LESNA, I., SABELIS, M. W. \& BURONFOSSE, T. 2009b. Molecular phylogenetic assessment of host range in five Dermanyssus species. Exp Appl Acarol, 48, 115-42.

46. ROY, L., GIANGASPERO, A., SLEECKX, N. \& ØINES, Ø. 2021. Who is Dermanyssus gallinae? Genetic Structure of Populations and Critical Synthesis of the Current Knowledge. Front Vet Sci, 8, 650546.

47. ROZAS, J., FERRER-MATA, A., SANCHEZ-DELBARRIO, J. C., GUIRAO-RICO, S., LIBRADO, P., RAMOS-ONSINS, S. E. \& SANCHEZ-GRACIA, A. 2017. DnaSP 6: DNA Sequence Polymorphism Analysis of Large Datasets. Mol. Biol. Evol., 34.

48. SCHICHT, S., QI, W. H., POVEDA, L. \& STRUBE, C. 2014. Whole transcriptome analysis of the poultry red mite Dermanyssus gallinae (De Geer, 1778). Parasitology, 141, 336-346.

49. SLATKIN, M. 1975. Gene flow and selection in a 2-locus system. Genetics, 81, 787-802.

50. SLATKIN, M. 2008. Linkage disequilibrium-understanding the evolutionary past and mapping the medical future. Nat Rev Genet, 9, 477-85.

51. SPARAGANO, O., PAVLICEVIC, A., MURANO, T., CAMARDA, A., SAHIBI, H., KILPINEN, O., MUL, M., VAN EMOUS, R., LE BOUQUIN, S., HOEL, K. \& CAFIERO, M. A. 2009. Prevalence and key figures for the poultry red mite Dermanyssus gallinae infections in poultry farm systems. Exp Appl Acarol, 48, 3-10.

52. SPARAGANO, O. A., GEORGE, D. R., HARRINGTON, D. W. \& GIANGASPERO, A. 2014. Significance and control of the poultry red mite, Dermanyssus gallinae. Annu Rev Entomol, 59, 447-66.

53. VALIENTE MORO, C., DE LUNA, C. J., TOD, A., GUY, J. H., SPARAGANO, O. A. \& ZENNER, L. 2009. The poultry red mite (Dermanyssus gallinae): a potential vector of pathogenic agents. Exp Appl Acarol, 48, 93-104.

54. VAN EMOUS, R. 2017. Verwachtte schade bloedluis 21 miljoen euro [Online]. Available: https://www.pluimveeweb.nl/artikel/163578-verwachtte-schade-bloedluis-21-miljoen-euro/ [Accessed 31 Jan 2020].

55. WRIGHT, H. W., BARTLEY, K., HUNTLEY, J. F. \& NISBET, A. J. 2016. Characterisation of tropomyosin and paramyosin as vaccine candidate molecules for the poultry red mite, Dermanyssus gallinae. Parasit Vectors, 9, 544.

56. ZENNER, L., BON, G., DERNBURG, A., LUBAC, S. \& CHAUVE, C. 2003. Preliminary studies of the monitoring of Dermanyssus gallinae in free-range poultry farms. Br Poult Sci, 44, 781-2.

\section{Figures}




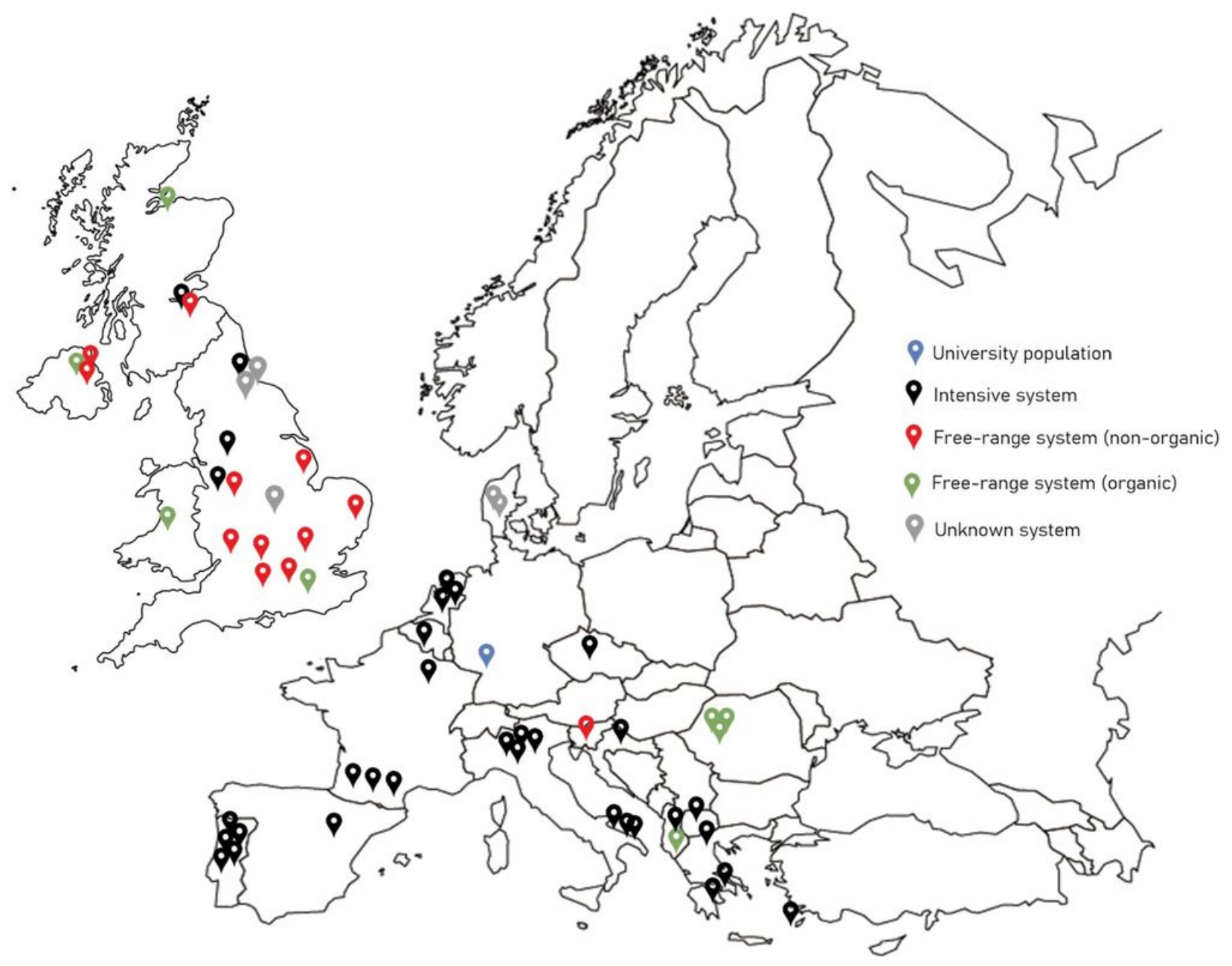

Figure 1

Approximate location and characteristics of farms sampled for Dermanyssus gallinae across the United Kingdom and from 15 mainland European countries. Key provided outlines production system utilised by farm. One UK farm provided no address so has not been mapped directly. Free-range system encompasses free-range layer farms and backyard poultry flock. 


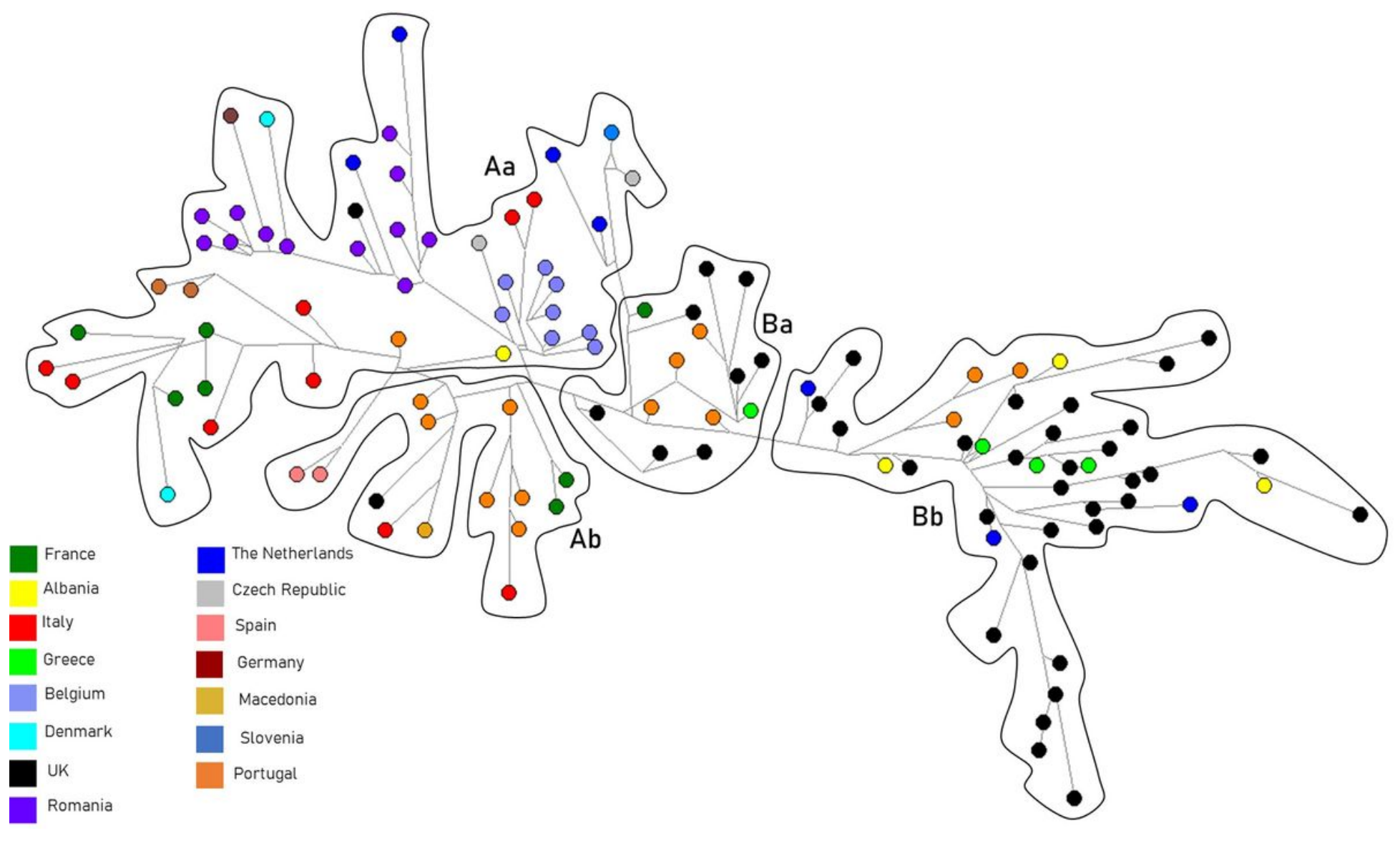

Figure 2

Network analysis of 117 pooled D. gallinae samples from the UK and the rest of Europe genotyped by NGS multiplex sequencing. Countries are colour coded in the key. Median-joining tree analysis was completed using Network 10.0.0.0. A total of 144 SNPs were used in this analysis. Clades $\mathrm{Aa}, \mathrm{Ab}, \mathrm{Ba}$ and $\mathrm{Bb}$ identified through ML phylogeny (as outlined in Figure 3) are indicated. 


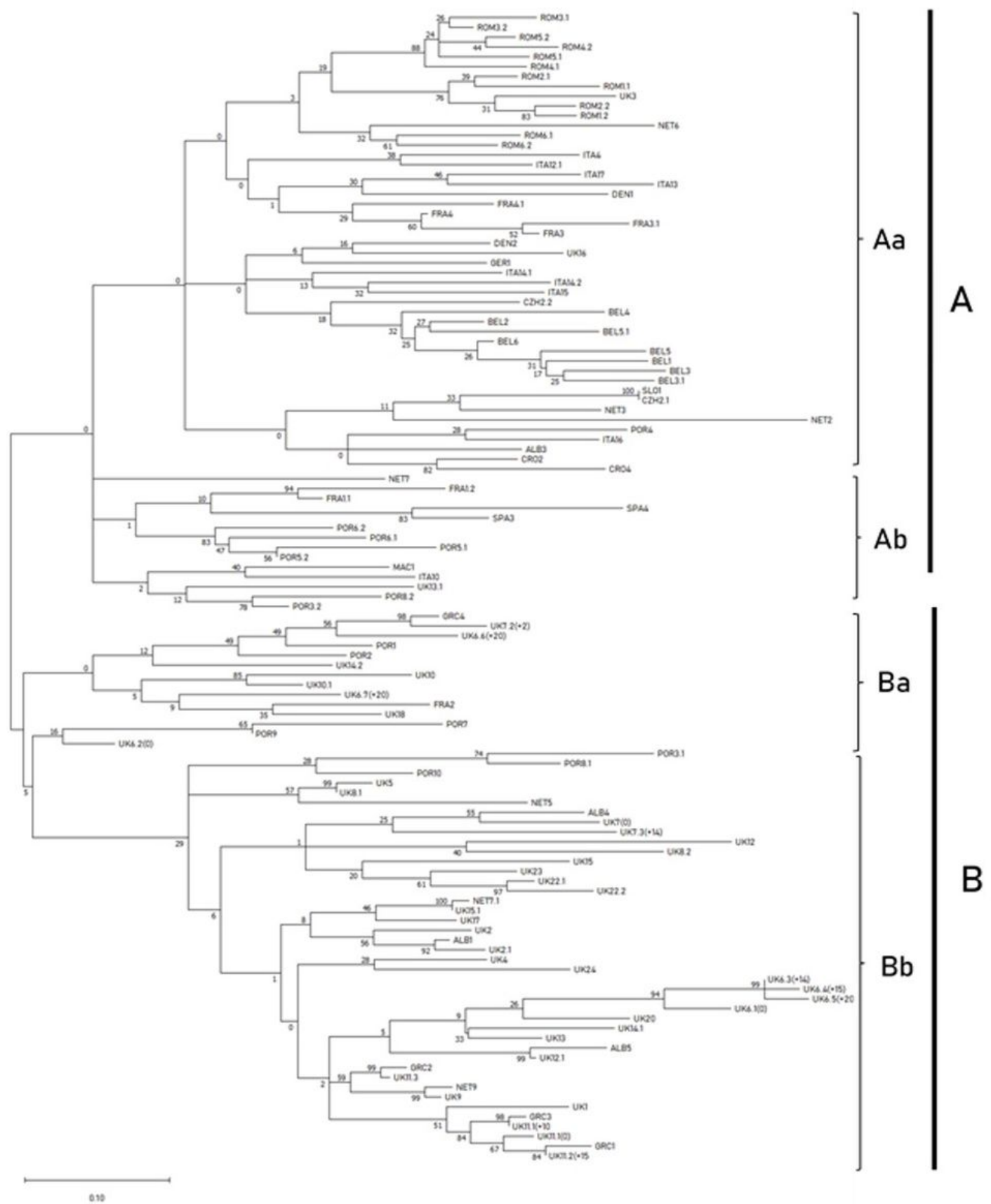

Figure 3

Phylogenetic tree of 117 pooled D. gallinae samples genotyped by NGS multiplex sequencing. Inferred using the GeneralTime Reversible parameter and maximum-likelihood with 1000 replicates. A gamma distribution was utilised to model evolutionary differences (shape parameter $=0.5$ ). A total of 144 positions were used in the analysis, encoding 117 nucleotide sequences. All evolutionary analysis was completed with MEGA X(40). 


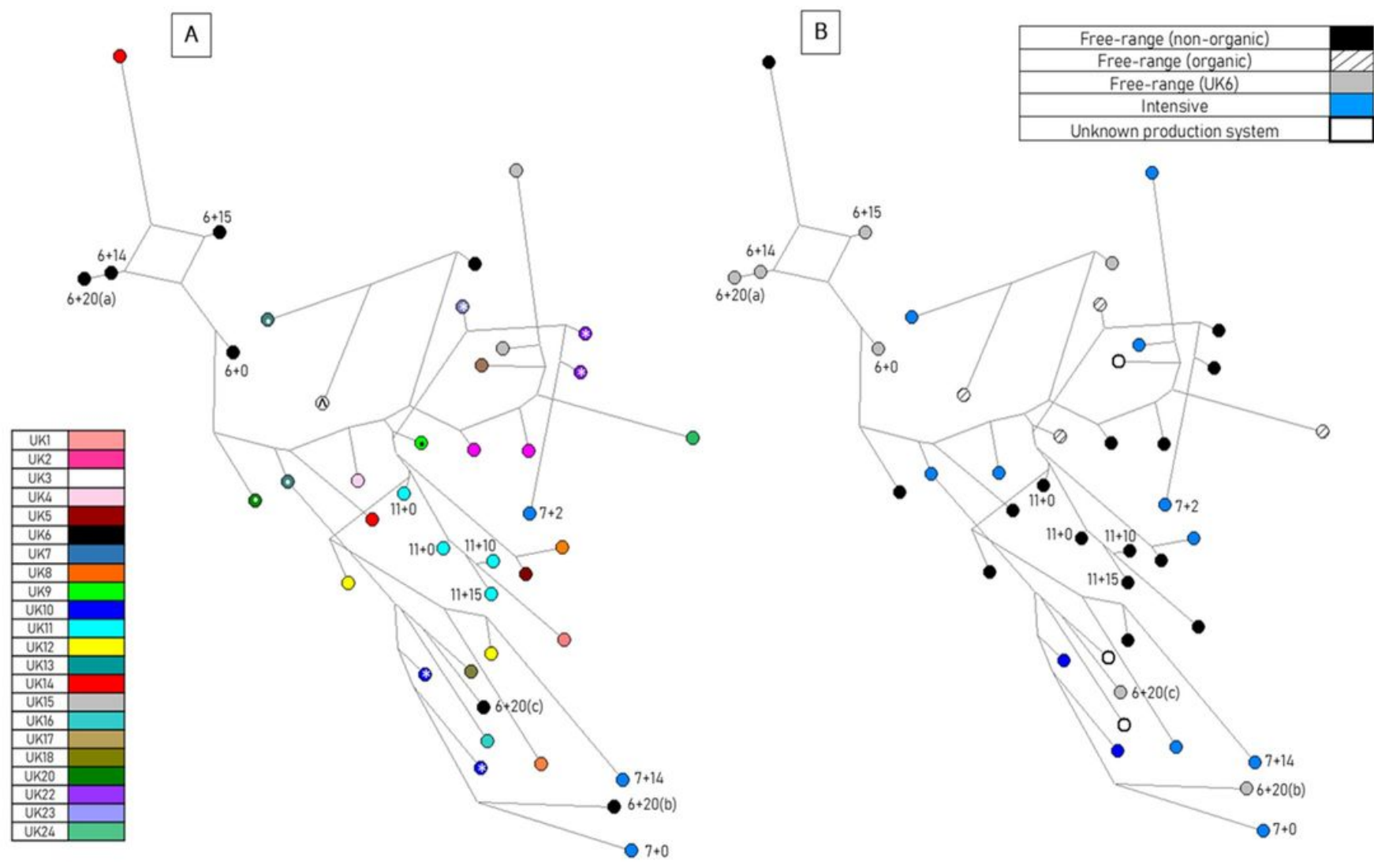

Figure 4

Network analysis of 41 pooled D. gallinae samples from the UK genotyped by NGS multiplex sequencing by farm (A) and production system (B). Clade two as identified in Figure 3 is indicated by the dashed line. Individual farms are colour coded in the keys provided, with countries designated by Northern Ireland $\left(^{*}\right)$ Scotland $(\cdot)$ and Wales $\left(^{\wedge}\right)$ and no symbol for English farms (A). Production systems are colour coded in the key (B). Farms UK6, UK7 and UK11 were sampled on multiple occasions, permitting analysis of temporal variation, where the date of visit is indicated by +0 for the first visit and the subsequent gap in months indicated. Multiple barns sampled for UK6 are indicated by letters (a) barn 1 (b) barn 2 and (c) barn 3. Median-joining tree analysis was completed on Network 10.0.0.0. A total of 144 positions were used in this analysis, encoded within 41 nucleotide sequences.

\section{Supplementary Files}

This is a list of supplementary files associated with this preprint. Click to download.

- 484079619250466943670485659418332231630848n.jpg

- SupplementaryFiles.docx

- SupplementaryTables.docx 